\title{
Effects of age and smoking on intima of muscular pulmonary arteries
}

\author{
JUNE M FERNIE, ${ }^{*}$ D LAMB $\dagger$ \\ From the *Institute of Occupational Medicine, and the $†$ Department of Pathology, University of Edinburgh \\ Medical School, Edinburgh, Scotland
}

SUMMARY The lungs of twenty three subjects, 12 non-smokers and 11 smokers, age range 19-81 years, were studied. All were free from cardiopulmonary disease. The cross sectionally cut muscular pulmonary arteries of each subject were measured in sections obtained from 12 representative tissue blocks using a digitiser. Intimal area was measured and artery size defined as total length of internal elastic lamina (IEL). Intimal abnormality was expressed in the form of an intima index calculated by dividing intimal area by the area enclosed by the IEL in its theoretically unwrinkled state. Mean intima indices were calculated for arteries in four size groups: $\leqslant 600 \mu \mathrm{m}, 601-1200 \mu \mathrm{m}$, $1201-1800 \mu \mathrm{m}$ and $>1800 \mu \mathrm{m}$ length of IEL.

For all subjects intimal abnormality was most severe in the smallest muscular pulmonary arteries; values for mean intima indices decreased with increasing size of artery. There were no lobar differences. The amount of intimal abnormality increases with age in all sizes of artery: in the $600 \mu \mathrm{m}$ size group this could average a $32 \%$ reduction in lumen calibre in those aged 60 or more. We were unable to detect any correlation between intimal abnormality and the subject's smoking history.

Those who study the pulmonary vascular bed are interested in determining what structural changes age and smoking bring about in its component vessels, particularly the muscular pulmonary arteries which form one of its most reactive parts by virtue of their role in regulating vascular resistance. In particular there is a need to establish "base line" values so that the effects of disease may be accurately assessed.

In the muscular pulmonary arteries the most common age change is that of intimal fibrosis, the prevalence of which increases dramatically with age, although there is considerable individual variation in its presence and extent. ${ }^{1-3}$ Most researchers have specifically stated that these intimal age changes are most common and most severe in the medium or smaller sized arteries ${ }^{34}$ and also that the changes tend to occur in the upper lobes. ${ }^{35}$ In addition to aging, smoking has been implicated as a cause of increased intimal thickening in muscular pulmonary arteries. ${ }^{56}$ All previous studies, however, have used methods of measurement - either simple grading systems ${ }^{6}$ or, more commonly, the "percentage intimal thickness" methods ${ }^{13-5}$ which are open to criticism, mainly on the grounds that the measurements of both the intima and artery size are affected by post mortem collapse or constriction of arteries.

The purpose of the present study was to quantitate

Accepted for publication 11 June 1986 
METHOD OF MEASUREMENT

Histological sections were scanned and each cross sectionally cut muscular pulmonary artery measured using the Graphic Digitising Systems 1 equipment. ${ }^{7}$ Arteries possessing a well defined internal elastic lamina ("digitisable") were measured using our first reported technique. ${ }^{7}$ Arteries in which the internal elastic lamina was ill defined ("undigitisable") were measured using a modification of that technique, the description and validation of which is given elsewhere. ${ }^{8}$ Measurements of intimal area and artery size were thus obtained for both "digitisable" and "undigitisable" arteries.

After measuring each artery ("digitisable" or "undigitisable") the degree of crinkling in its internal elastic lamina (crinkle grade) was estimated by eye on a scale of $0 \rightarrow 4$, indicating none to intense constriction or collapse. ${ }^{8}$

\section{CALCULATION OF INTIMA INDICES}

Intima indices were calculated by dividing the area of the intima by the area enclosed by the internal elastic lamina in its theoretically unwrinkled state, ${ }^{7}$ which is necessary to accommodate differences in the degree of collapse or constriction between arteries. Values for intima index range from $>0$ to $\leqslant 1$, indicating minimal through to total occlusion of the artery lumen by intimal change.

\section{ANALYSIS OF DATA}

The individual artery data and the data on age and smoking habit were transferred to tape on the main frame computer (a Prime 750) at the Institute of Occupational Medicine. All analyses were carried out using the statistical package Minitab. ${ }^{910}$ As it can only carry out linear regressions the linearity of each regression line was checked. In instances where the fit was not linear then the data were reanalysed using the statistical program Simple Regressions ${ }^{7}$ in conjuction with the digitiser set up.

\section{PRELIMINARY ANALYSES OF ARTERY DATA} FOR INDIVIDUAL SUBJECTS

We have shown ${ }^{7}$ that subjects are best compared by calculating mean intima indices for arteries subdivided into size groups. The purpose of the preliminary analyses was to determine what these size groups should be. An additional aim was to establish whether it was necessary to account for different classes of artery separately, the classes in question being "digitisable" and "undigitisable" cross sectionally cut arteries and arteries from different lobes.

Intima indices were calculated and plotted against total length of internal elastic lamina for all measured arteries of each subject using Minitab.
Results

\section{SUMMARY DATA FOR INTIMA}

For all subjects the relation between intima index and artery size was broadly similar, the value for intima index tending to increase with decreasing size of artery, which confirms our earlier findings ${ }^{7}$ (fig 1). There were no consistent or obvious differences between "digitisable" and "undigitisable" arteries. Fig 2 illustrates that there were no consistent lobar differences either.

The decision on what size groups to use for the calculation of mean intima indices was made with a view to satisfying these two criteria: an acceptable number of arteries in each group, and cut offs at points where the relation between intima index and artery size seemed to be changing. The following size groupings were thought to provide the best possible compromise: $\leqslant 600 \mu \mathrm{m}, 601-1200 \mu \mathrm{m}, 1201-1800 \mu \mathrm{m}$ and $>1800 \mu \mathrm{m}$ total length of internal elastic lamina, although for some subjects there was no information for the largest size group. For each subject mean intima indices of arteries in these size groups were calculated and are referred to as II600, III 200, III800, and II $>1800$, respectively, the figures denoting the upper limit of the size range of arteries were included in each group.

THE EFFECTS OF AGE AND SMOKING ON THE INTIMAL COMPONENT OF MUSCULAR PULMONARY ARTERIES

Using Minitab the mean II600, II1200, II1800, and II $>1800$ values were plotted against age for the



Fig 1 Relation between intima index and artery size for case 16. "Digitisable" ( ) and "undigitisable" ( $\Delta$ ) cross sectionally cut arteries separately identified. $\phi=$ coincident points. 




Fig 2 Relation between intima index and artery size for case 16. Upper ( ) and lower $(\triangle)$ lobe arteries separately identified. $\phi=$ coincident points.

whole group, separately identifying the non-smokers and smokers.

Taking the group as a whole it was found that for all four size groups of muscular pulmonary arteries the mean intima index increased with increasing age, although not always in a linear fashion. Only for the 600 (fig 3) and 1200 (fig 4) size groups was the increase significant $(\mathrm{p}<0.01$ and $\mathrm{p}<0.05$, respectively).

The range of mean intima indices observed at different ages was usually quite considerable and most evident in the smaller arteries. In the smallest size group (fig 3 ) the mean intima indices for subjects less than 40 years old covered a narrow range 0.03 to 0.07 . There was little, if any, change until the mid to late 50 s by which time the range had extended to almost $0 \cdot 15$. The greatest variation, however, was

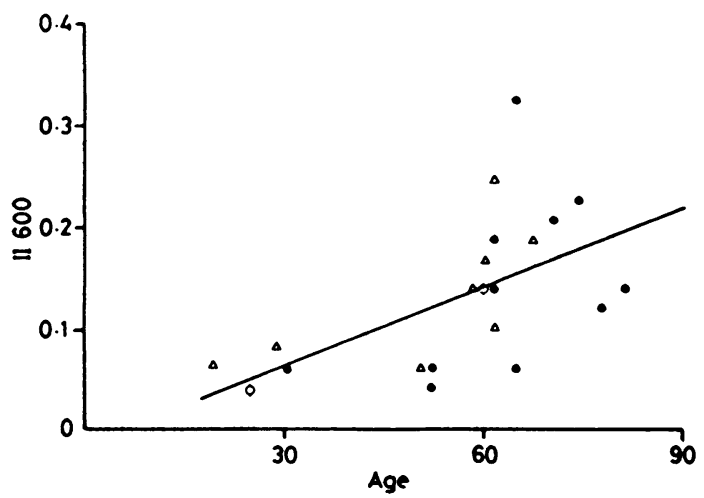

Fig 3 Relation between mean intima index 600 and age. Smokers $(\triangle)$ and non-smokers $(\bigcirc)$ separately identified. $\phi=$ coincident points. Line of best fit is: $y=-0.018+$ $0.003 x, r=0.61$.

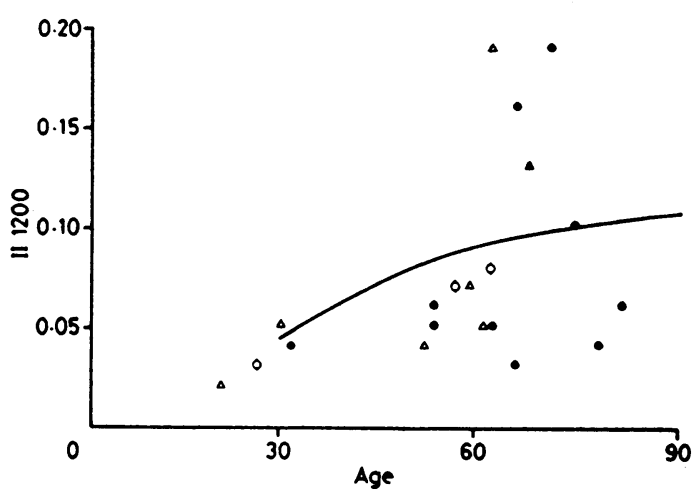

Fig 4 Relation between mean intima index 1200 and age. Smokers $(\triangle)$ and non-smokers $(\bigcirc)$ separately identified. $\phi=$ coincident points. Line of best fit is: $y=-0.016+$ $0.06 . \log _{\mathrm{e}} x, r=0.49$.

seen in the age group 60 plus, with some subjects showing mean II600 values that were of the same order as those seen in subjects aged 40 or less, while at the other extreme there were subjects whose muscular pulmonary arteries showed a degree of intimal abnormality that, on average, amounted to a $32 \%$ reduction in lumen calibre.

A similar picture was observed for the II1200 values (fig 4), the greatest variation being seen in the 60 plus age group. At any age the mean II1200 values (overall range 0.02 to 0.19 ) were less than the II600 values (overall range 0.03 to 0.32 ), indicating a less severe increase in intima with increasing size of artery. This trend continued and became even more noticeable; in the 1800 group the maximum mean intima index observed was 0.08 , whereas in the $>1800$ size group it was only 0.04 .

A comparison of similarly aged smokers and nonsmokers in figs 3 and 4 suggested that there was little difference, if any, between these two groups with respect to II600 and II1200 values. Similar trends were evident with the III800 and II > 1800 values (result not illustrated).

Further investigation of the effect of smoking on the intima was confined to subjects aged $50-70$ years, this being the only age band in which adequate numbers of smokers and non-smokers were available for comparison. The table shows the mean intima indices

Table Mean (SD) intima indices for smokers and non-smokers aged 50-70 years

\begin{tabular}{lllll}
\hline & $I I 600$ & $I I 1200$ & $I I 1800$ & $I I>1800$ \\
\hline Smokers & $0.14(0.05)$ & $0.09(0.05)$ & $0.05(0.02)$ & $0.02(0.005)$ \\
Non-smokers & $0.14(0.10)$ & $0.09(0.06)$ & $0.04(0.02)$ & $0.02(0.02)$ \\
\hline
\end{tabular}


for smokers and non-smokers within this age band. No differences were detected between the two groups.

\section{COMPARISON OF THE CRINKLE GRADE OF}

ARTERIES FROM DIFFERENT LOBES

Mean crinkle grades were calculated for the upper and lower lobe arteries of each subject. These 23 sets of mean values were then subjected to a paired $t$ test, which indicated a highly significant $(\mathrm{p}<0.002)$ difference between the crinkle grade of upper and lower lobe arteries, those of the upper lobe being much more crinkled (constricted or collapsed).

\section{Discussion}

The intima has received less attention than the media in studies of "undiseased" subjects, which is a reflection of the importance most pathologists place, perhaps mistakenly, on medial hypertrophy in disease states and its assumed links with functional changes in the pulmonary circulation. As small changes in vessel calibre due to intimal thickening would have a disproportionately large effect on pulmonary vascular resistance, however, it may be that the importance of intimal abnormality has been greatly underestimated.

Using measuring techniques that are unaffected by artery collapse, or constriction, or the generally irregular distribution of intimal abnormality, the present study has substantiated previously reported findings that the amount of intimal abnormality increases with increasing age, ${ }^{131112}$ although at any age there is considerable individual variation in the amount of abnormality present, particularly in older subjects. ${ }^{13} 13$

Although it has been stated that the extent of intimal abnormality associated with aging is much less prominent in the larger muscular pulmonary arteries, ${ }^{3411}$ the present study has provided an accurate measure of what that extent is; in those arteries measuring greater than $1200 \mu \mathrm{m}$ length of internal elastic lamina the extent of intimal abnormality never amounts to more than an average reduction in lumen calibre of $10 \%$, even in very old subjects. This is unlikely to be of any functional importance to the pulmonary circulation.

In contrast, the extent of intimal abnormality in the very small muscular pulmonary arteries increases noticeably with increasing age-beyond 40 to 45 . Although there is a considerable individual variation in the mean intima index of arteries in this size group, the extent of intimal abnormality can amount to as much as a $32 \%$ reduction in lumen calibre in those aged 60 or more. There are several separate points that need to be made here. Firstly, these results corroborate other workers' findings of mean percentage intimal thickness (percentage of internal diameter) values in the order of $20 \% .^{35}$ Secondly, it seems unlikely that a mean effective reduction in lumen calibre of $32 \%$ in the very small arteries of some subjects would not cause some functional changes in the pulmonary circulation as a whole, especially when one considers that the value of $32 \%$ is a minimum possible value, the intima index being a measure of intimal abnormality or lumen occlusion, which is independent of the degree of active constriction present in the artery. Thirdly, the present study has established accurately the range of values one would expect to see in "undiseased" subjects in the very small muscular pulmonary arteries, which are most affected by intimal thickening. The fact that this range is quite extensive, particularly in older subjects, will undoubtedly affect the assessment and interpretation of intimal abnormality in disease states.

The main thrust of previous studies of the pulmonary arteries of "undiseased" subjects has been to elucidate the effects of aging, relatively little attention having been paid to the effects of smoking.

In the present study accurate and extensive data were available on smoking histories, and our results gave no indication that smoking has a direct effect on the intima, at least in those aged 70 or less. Others have concluded that smoking does have an effect as smokers show an increased intimal thickness ${ }^{5}$ or an overall increase in the thickness of the walls of the small arteries. ${ }^{6}$ Both these studies, ${ }^{56}$ however, may be criticised on the grounds that the methods used could not have accurately assessed either the extent of intimal abnormality or artery size.

In our study the age ranges of the non-smokers and smokers were different, and we consider that it would be useful to apply our measuring techniques to the muscular pulmonary arteries of some old (more than 70) smokers; this should clarify the long term effects of smoking, if any.

The main contribution made by the present study is the production of accurate information on the amount and range of intimal abnormality seen in a group of "undiseased" subjects of different ages and smoking habits. We consider, however, that an additional and very useful contribution the study has made is to dispel a long held belief on the part of many workers ${ }^{35}$ that intimal abnormality tends to occur in the upper lobes of the lung. Admittedly, pathologists have been puzzled as to why this should be so: Wagenvoort in particular has stated, "it is hard to explain this unexpected finding." 3 Several theories have been put forward but the most commonly held opinion is that it results from differences in blood flow in the upper and lower lobes of the lung, the reduced blood flow in the apex causing an increased tendency to thrombosis in periods of lung infections etc. ${ }^{313} \mathrm{We}$ have shown that there is no need for these complex 
theories; quite simply there are no differences between lobes in "undiseased" persons, in terms of amount of intima, but arteries from the upper lobes seem to be more constricted or collapsed than those from the lower lobes. It is this latter factor, coupled with a measuring technique, which is not independent of the degree of collapse or constriction present, that has resulted in the conclusions drawn by other workers that the per cent intimal thickness is greater in arteries from the upper lobes. ${ }^{35}$

We would like to thank Andy Douglas of the Pathology Branch, Institute of Occupational Medicine for helping with the transfer of data on to the main frame computer. Our thanks also go to the Pathology Branch technical staff for their help with the illustrations.

This study was funded by the National Coal Board.

\section{References}

1 Brenner O. Pathology of the vessels of the pulmonary circulation. Part II. Secondary pulmonary vascular sclerosis. Arch Intern Med 1935;56:724-52.

2 Harris P, Heath D. The human pulmonary circulation. 2nd ed. Edinburgh: Churchill Livingstone, 1977.
3 Wagenvoort CA, Wagenvoort $\bar{N}$. Age changes in muscular pulmonary arteries. Archives of Pathology 1965;79:524-8.

4 Semmens $M$. The pulmonary artery in the normal aged lung. Br J Dis Chest 1970;64:65-72.

5 Hale KA, Niewoehner DE, Cosio MG. Morphologic changes in the muscular pulmonary arteries: relationship to smoking, airway disease, and emphysema. Am Rev Respir Dis 1980; 122:273-8.

6 Auerbach O, Short AP, Hammond EC, Garfinkel L. Smoking habits and age in relation to pulmonary changes. Rupture of alveolar septums, fibrosis and thickening of small arteries and arterioles. New Engl J Med 1963;269:1045-54.

7 Fernie JM, Lamb D. New method for measuring intimal component of pulmonary arteries. J Clin Pathol 1985;38:1374-9.

8 Fernie JM, Lamb D. Method for maximising measurements of muscular pulmonary arteries. J Clin Pathol 1985;38:1380-7.

9 Ryan TA, Joiner BL, Ryan BF. Minitab reference manual. Boston: Duxbury Press, 1982.

10 Ryan TA, Joiner BL, Ryan BF. Minitab student handbook. Boston: Duxbury Press, 1982.

11 Warnock ML, Kunzmann A. Changes with age in muscular pulmonary arteries. Arch Pathol Lab Med 1977;101:175-9.

12 Wagenvoort CA, Wagenvoort N. Pathology of pulmonary hypertension. New York: John Wiley and Sons, 1977.

13 Wagenvoort CA, Wagenvoort N. Pulmonary vascular bed. Normal anatomy and response to disease. In: Moser KM, ed. Pulmonary vascular diseases. New York: Marcel Dekker, 1979.

Requests for reprints to: June M Fernie, Institute of Occupational Medicine, 8 Roxburgh Place, Edinburgh EH8 9SU, Scotland. 